\title{
QUALITY ASPECTS OF AERIAL DIGITAL ORTHOPHOTOS, THE PRODUCERS POINT OF VIEW
}

\author{
A. Wiedemann ${ }^{\text {a }}$, S. Beckmann ${ }^{\mathrm{b}}$ \\ ${ }^{\text {a }}$ FH Erfurt, University of Applied Science - albert.wiedemann@fh-erfurt.de \\ b BSF Swissphoto, Mittelstr. 7, D-12529 Schönefeld, Germany - sandra.beckmann@bsf-swissphoto.com
}

Commission II, WG II/6

KEY WORDS: Orthoimage, Quality

\begin{abstract}
:
The paper gives an overview on the problems occurring during commercial digital orthophoto (DOP) production. There is a wide variety in requirements on orthophotos. It is no problem to produce good orthophotos in small areas, whereas production of good and homogeneous orthophotos in large areas remains a challenge. Therefore, we discuss large area orthophotos based on regular areal flight with regular flight parameters and nadir images, not on large numbers of images creating orthophotos based on various oblique images.

The focus of the paper is to optimize the process of line production of orthophotos to achieve the best results under minimum usage of resources. Beside improvements in commercial software, the improvement in process design can help to achieve this objectives. It is necessary the attach importance to the different quality aspects to invest the limited resources at the most profitable process step.

The requirements on orthophotos can be grouped into three categories: geometric, radiometric and aesthetic requirements. The main intention is to generate correct and well interpretable orthoimages, putting attention to geometry and radiometry. Many requirements cause enormous expenditures in flight time, number of images and amount of interactive editing work.

Among the geometric aspects, the accuracy is the prominent factor. The geometric quality of the underlying digital terrain model (DTM) is of outstanding importance. Depending on the used focal length during acquisition and overlap, geometric errors in the DTM are directly represented in the orthophoto. Particularly at manmade surface break lines, even horizontal DTM errors occur striking in the orthophotos.

A special consideration is to put on objects outside the DTM surface. While buildings may be represented in a good manner in a digital surface models (DSM) to generate so called "true orthophotos", there is no way to represent vegetation, cables, poles etc. in an appropriate manner in any DSM.

Seam lines have to disappear in the mosaics. There should be no indication for the detection of the used seam lines nether in geometric nor in radiometric edges.

The radiometric aspect include a good distribution of the color values in the histogram, good contrasts should become quantified. It is impossible to measure the aesthetic quality, but is has to be homogeneous over the whole project area without eye-catching artefacts.

In the conclusion, we want to give to the producers of orthophotos and their clients a list of criteria and quality figures to be agreed on before the production starts to avoid later discussions.
\end{abstract}

\section{QUALITY OF ORTHOIMAGES}

\subsection{Function of Digital Orthophotos (DOPs)}

The basic idea of Digital Orthophotos (DOP) is the vertical (orthogonal) projection of images of 3D objects on a defined surface, typically a map projection. This is the most common method of geo visualization. Each position on the map projection is represented by a single colored pixel $(2.5 \mathrm{D}$ pixel is a function of location). Unfortunately, the surface of the earth is not a function, but a relation to the location. On some locations not only one surface, but several surfaces are available. On bridges, we have to represent only two surface, in vegetation the number of surfaces to be represented is outnumbered. It is generally accepted to represent the fractal surface by a single, numerical represented surface - the Digital Terrain Model (DTM) or the Digital Surface Model (DSM) (Fig.1). If the surface representation is quite steep, it might come to occluded areas in the images. To avoid them a dense flight pattern might become necessary.

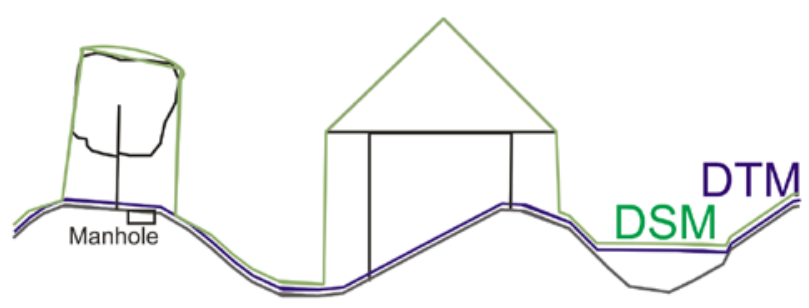

Fig. 1: DTM and DSM

\subsection{Generation of DOPs - Process Chain}

The whole process chain of orthophoto production consists of planning, flight, orientation and orthophoto production including quality checks. The used model (DTM or DSM) may

\footnotetext{
* Corresponding author
} 
result from a separate source or at least a separate process, which is not covered in this article (Wiedemann \& Peter 2006). The process chain for the complete orthophoto production is described below (Fig. 2).

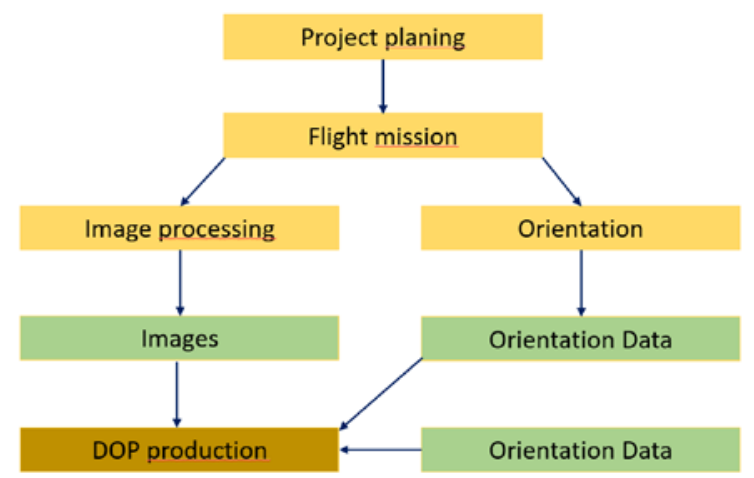

Fig. 2: Process steps complete DOP process

The DOP production in the office begins with already oriented aerial images, an existing terrain/surface representation, a defined map projection and tiling for the DOP to be generated. The target is to generate a geo visualization for a defined area, represented by a given perimeter (Fig. 3).

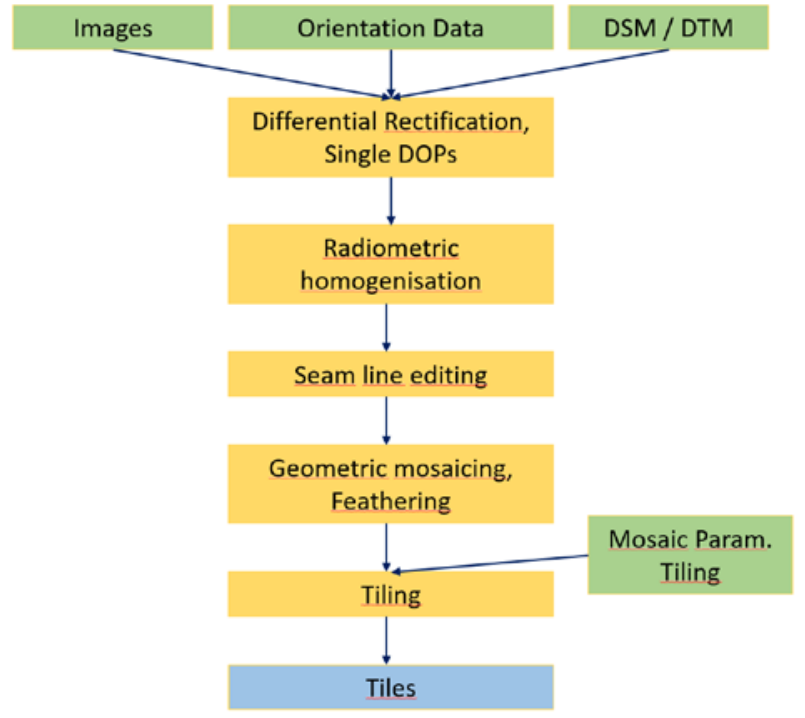

Fig. 3: Process chain for DOP production

\subsection{Quality Aspects of Digital Orthophotos}

Some national regulation exist on the quality of Digital Orthophotos (e.g. in Germany AdV 2012, DIN 18740-3). There are three factors to be concerned, when we discuss the quality of digital orthophoto (DOP): first of all, they have to be correct - in sense of geometry and radiometry, according to the definition and requirements of purchaser of the orthophotos. For geo visualization and earth sciences, the earth surface is in the center of attention, therefore the differential rectification should be done to the Digital Terrain Model (DTM), representing the modeled earth surface. For the analysis of roof surfaces, the differential rectification should be done to the Digital Surface Model (DSM), representing the highest points when surfaces are closed. Many objects in the reality cannot be described with closed surfaces under a given image resolution. E.g. with $10 \mathrm{~cm}$ Ground Sampling Distance powerlines, cables, light poles, street signs and tree canopies cannot be described in a satisfying manner in a digital surface model. Per definitionem the surfaces should only represent static objects - no cars, no trucks and vegetation. Therefore, in most cases the use of the DTM is to be preferred - delivering a more homogeneous result. It has to be considered that a manhole below a tree without leafs can be located correctly in a DTM-Based orthophoto, but not in a DSM-based orthophoto.

The second quality aspect is readability - is it possible to determine the local situation based only on the orthophoto? We use orthophotos to collect information on an environment without being on site. The situation in the orthophoto is frozen to the date of image acquisition. Therefore, the age of the images is of high importance. Sufficient contrast, natural colors and a representative appearance of the site help to interpret the situation. Even objects, which should be removed by definition, are helpful for the interpretation. Parking cars show proof that the site is accessible by cars, the distribution of people show the accessibility by pedestrians.

The last quality factor is aesthetic appearance. The observer should not be distracted or irritated by unnatural artefacts and situations. Our human visual sensor system is highly sensitive to such images. Jumping roof have something like a magnetic effect to our eyes.

The sample in Fig. 4 is typical. Even if the orthophoto is correct here, our eye is attracted by the break of the real circular pattern resulting from the seam line between the buildings in the south. The broken circle is irritating, even when den DOP is done according to the state of the art, but based on a digital terrain model (DTM).

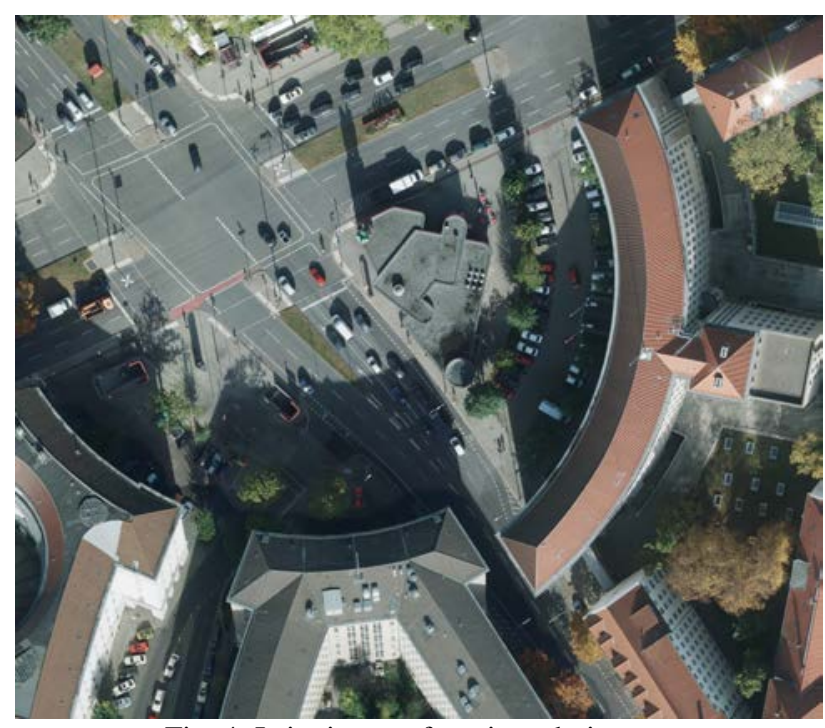

Fig. 4: Irritating artefacts in orthoimages

\subsection{Quality measurement}

It is difficult to benchmark the quality of digital orthophotos without normalized quality parameters. The geometric quality can be determined by comparison with geodetic data from other sources. The geometric quality is based on the quality of the used images orientation parameters (interior and exterior orientation), the maximum distance of used image parts from the nadir point and the horizontal and vertical accuracy of the used 3D model. The quality of the used orientation parameters 
can be evaluated using images chips (Fig. 5), by reprojection of geodetic measured checkpoints into the raw images. The quality of the orientation parameters is a premise for a good quality of the DOPs. BSF Swissphoto has developed image chips - a reprojection of geodetic measured check points in the oriented images to check the AT quality
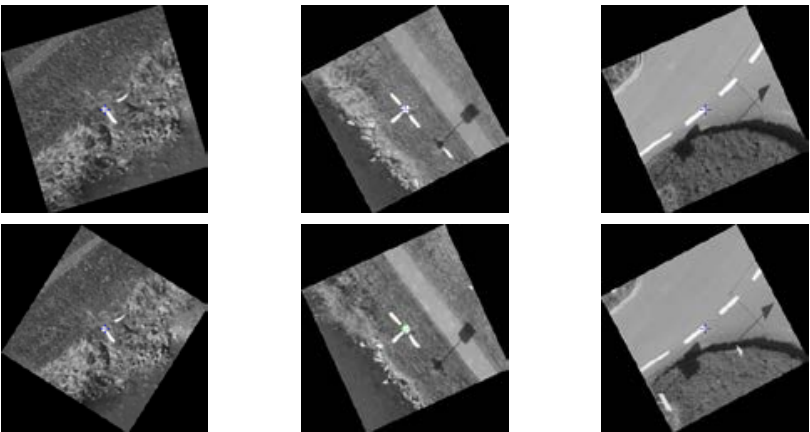

Fig. 5: Image chips (raw images) in oriented images

A check of the geometric quality of intermediate generated single DOPs before the mosaicking can be done by comparing the generated single DOPs in the overlapping zone (Fig. 6). The geometric accuracy can also be determined by detecting residues between two adjacent overlapping orthophotos. The single DOPs have to be checked by visual inspection: Are there unnatural deviations in curvature of lines (Fig. 8), are the luting due to steepness of the terrain (Fig. 10), or duplications?

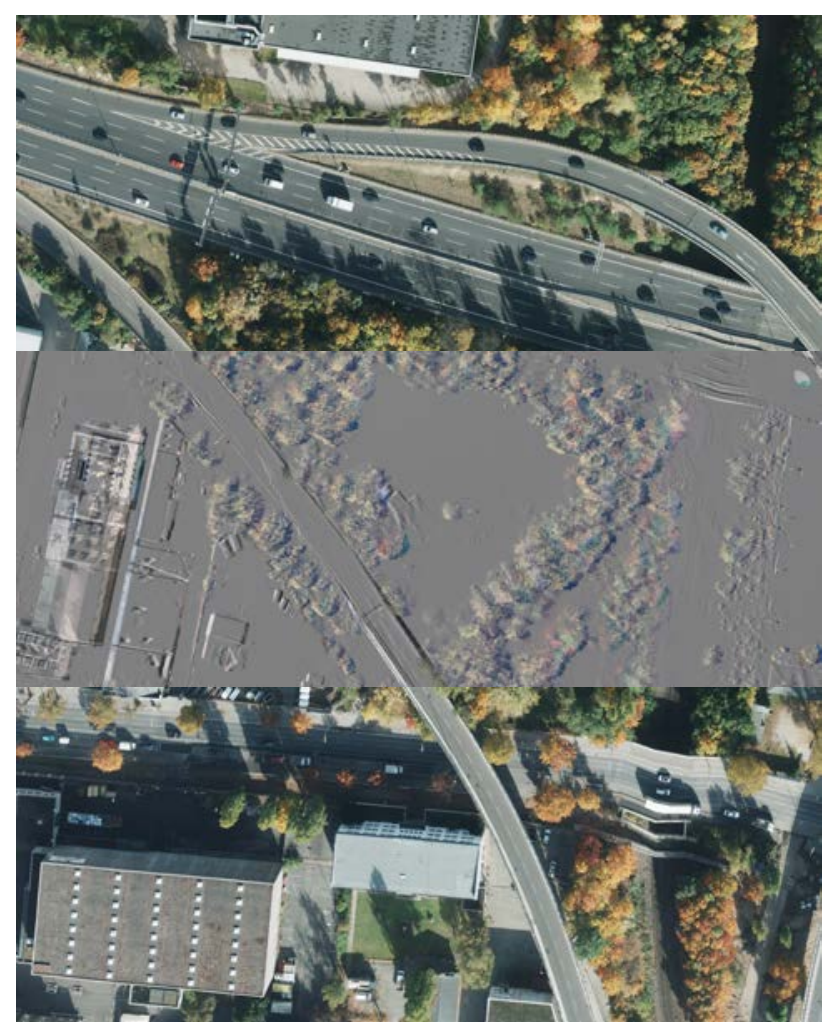

Fig. 6 Geometric quality by DOP difference in overlapping zones.

To check the geometric quality of DOP mosaics the best way is to superimpose any existing vector data (Fig. 7). In an ideal situation, the data result from an independent source. Other geometric aspects of the orthophoto mosaics are the questions: continuity of lines crossing the seam lines.

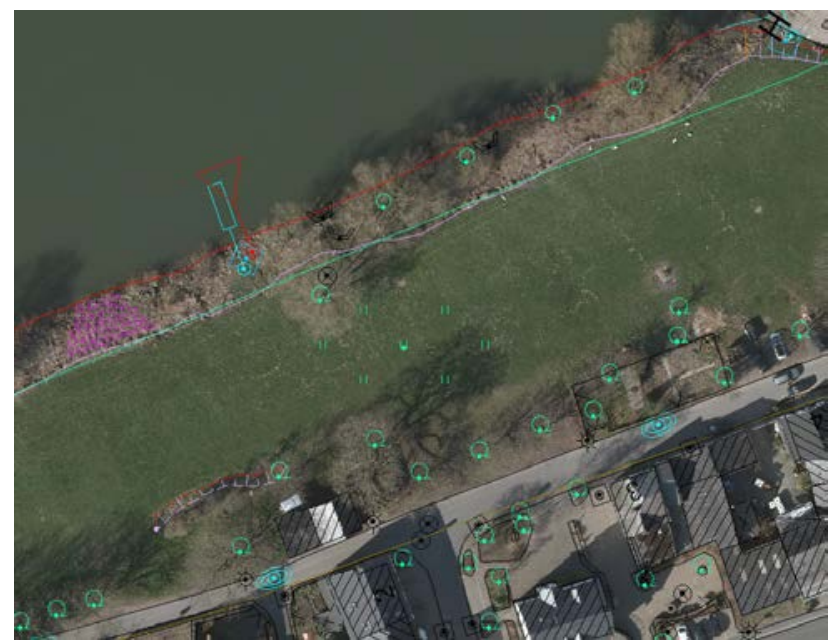

Fig. 7: Geometric quality check by superimposition of vector data

Unfortunately, there is no way to proof the readability of the orthophotos. The readability is subjective and depends on the background and experience of the user.

The radiometric quality can be determined by statistical values for the histogram of each color band of the orthoimages, the difference of the single band gray values of real world gray surfaces (asphalt, concrete, gravel). The following statistical values can be used to determine the quality: $\mu_{R}, \mu_{G}, \mu_{B} \sigma_{R}, \sigma_{G}$, $\sigma_{\mathrm{B}}, \Delta \mathrm{gW}-\mathrm{R}, \Delta \mathrm{gW}-\mathrm{G}, \Delta \mathrm{gW}-\mathrm{B}$.

\section{QUALITY PARAMETERS}

\subsection{Flight Parameters}

The flight parameters have a serious influence on the quality of the DOPs. The higher the flight level and the longer the focal distance the smaller the angles between rays, the closer the central perspective is similar to an orthogonal projection. The higher the long track and cross track overlap, the smaller the area to be used for the mosaicking. With a higher overlap the resulting geometric errors fade away, but the costs for flying explode (Table 1 ).

\begin{tabular}{|l|r|r|}
\hline & Regular Flight & Optimized Flight \\
\hline $\begin{array}{l}\text { Overlap (along / } \\
\text { cross) }\end{array}$ & $60 \% / 30 \%$ & $80 \% / 60 \%$ \\
\hline Flight time & $100 \%$ & $200 \%$ \\
\hline Images & $100 \%$ & $400 \%$ \\
\hline
\end{tabular}

Table 1: Increase of cost due to optimized flight parameters

\subsection{Geometric Aspects}

The geometric quality of the DOP is a function of the flight parameters, the quality of the sensor orientation, the distance of the pixel from the nadir point and finally the quality of the DTM/DSM. Due to a limited quality of the surface representation, some geometric errors remain in the DOP. It is the task of the orthophoto production to minimize the remaining errors and to hide their geometric influence. For this purpose the seam line editing has to be done carefully. The use of building data to avoid building cuts is here very helpful. 


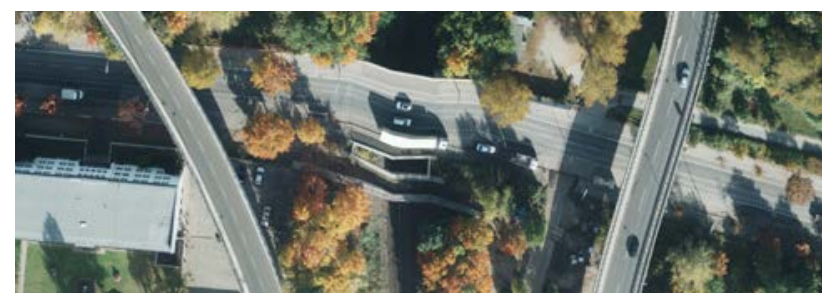

Fig. 8: Results of remaining DTM errors

\subsection{Radiometric Aspects}

The single images have to be of high quality. The radiometric quality aspects concerning the single images cover the brightness and contrast of the images, the distribution of color values in the histogram, the color balance.

The mosaic quality is focused on the homogeneity of the mosaic. In an optimized mosaic, the edges between the single images fade away. In some situations, it makes no sense to hide the seam lines, e.g. in non homogeneous water bodes.

\subsection{Additional Effects}

Sometime some additional effects may result if the processing does not avoid them. An example is the Moiré pattern, resulting e.g. down sampling of regular patterns, like roof tiles (Fig. 9).-

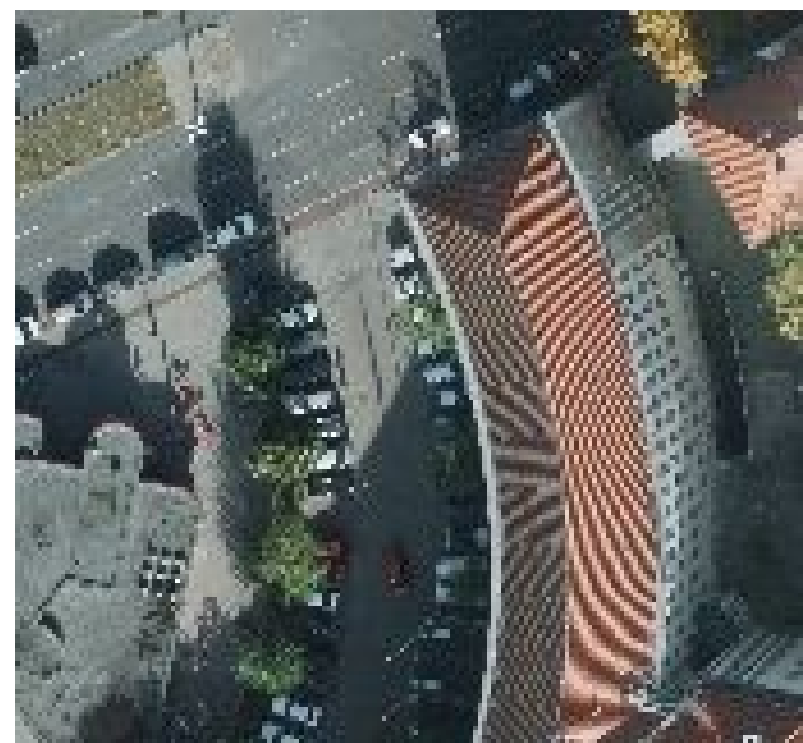

Fig. 9: Moiré Effects

In steep areas, the image ray is nearly parallel to the terrain surface it may come to luting (Fig. 10). This area occurs mainly in mountainous areas and can be avoided sometimes by taking the area out of an adjacent image. This process can be automated.

In water bodies it comes sometimes to special reflections. This is irritating, but an editing of water bodies makes in general no sense, but causes immense effort. Sometimes it simple to do so do it, but not in general. Seamlines remain visible when crossing wave fronts.

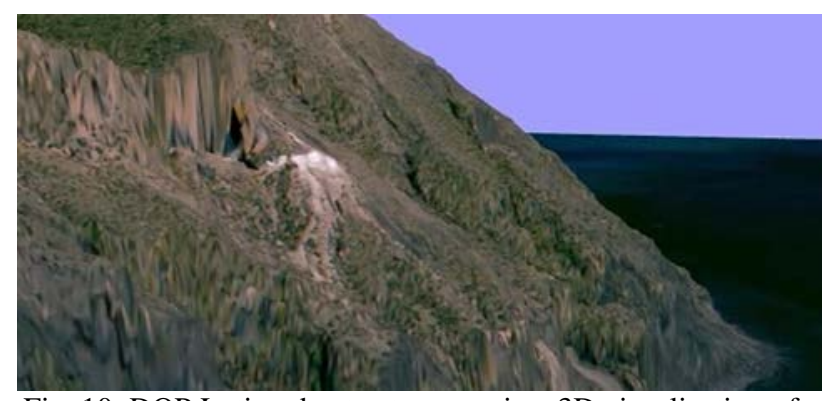

Fig. 10: DOP Luting due to steepness in a 3D visualization of a DOP

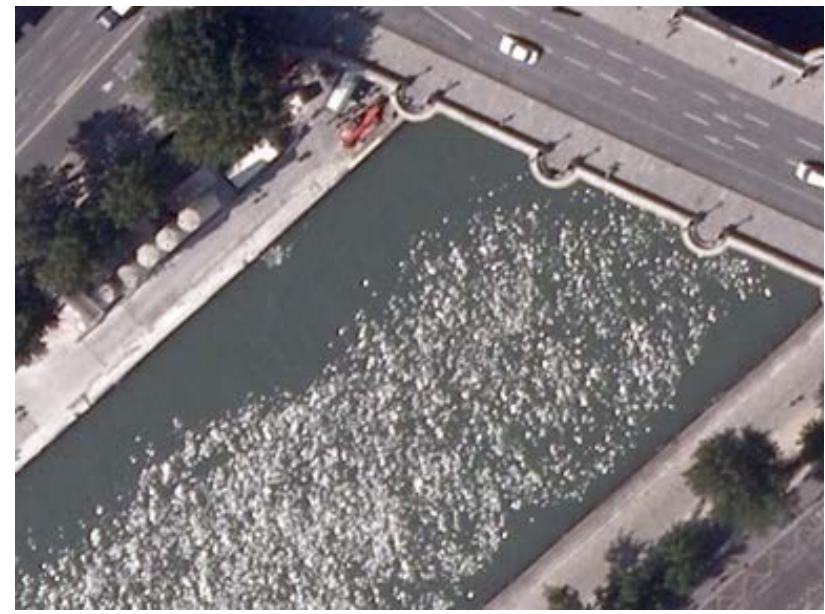

Fig. 11: Irritating wave reflections in water bodies

\subsection{True DOPs}

The remaining problem is based on the insufficient surface representation by a DSM to be used for so called true ortho photos. Irregular open structures like the Eifel Tower (Fig. 12) are mathematically represented by a closed surface, like a towel over the structure. This might be o.k. in some cases, like the canopies in a forest, but also might cause serious irregular results (Fig. 13)

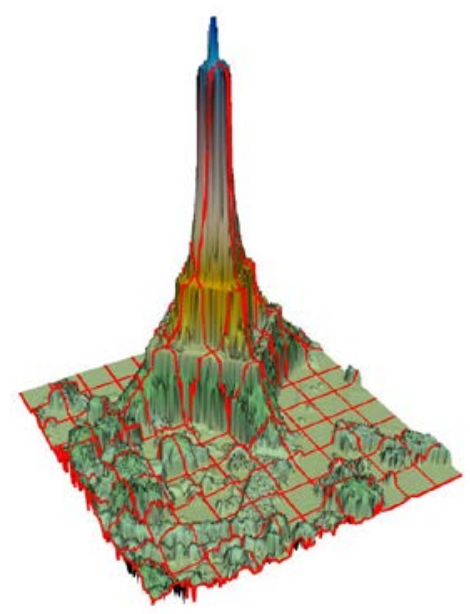

Fig. 11: DSM of the Eifel tower, generated from airborne laser scanning. 


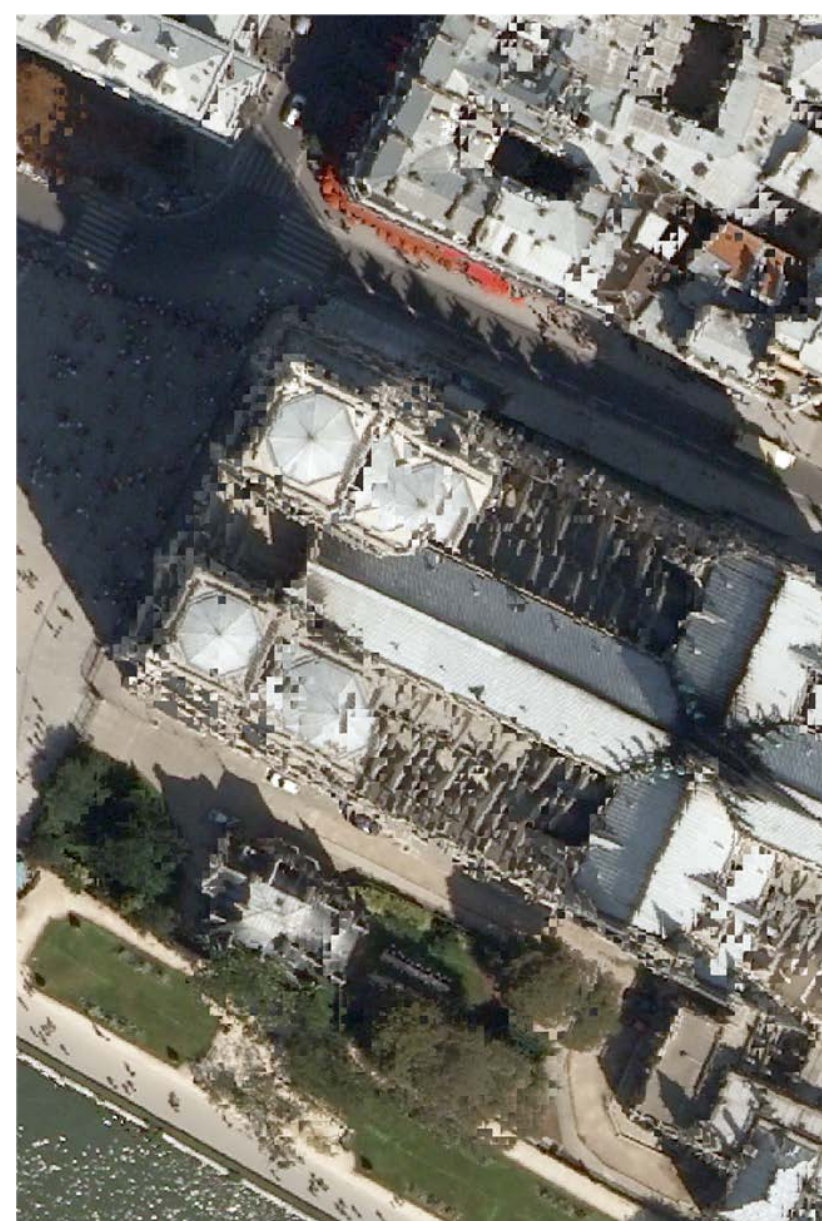

Fig. 12: True orthophoto on an irregular surface (gothic church roof with dublications etc.)

The requirement on the flight parameters, if a production of True DOPs is considered, are quite high: At least more than $50 \%$ cross track overlap to fill gaps behind rising buildings.

Due to the limited modeling of the surface, some artefacts and mistakes remain in the generated DOPs (Wiedemann \& Wicki 2010) (Fig. 12 and 13).

\subsection{Dense Matching}

Another new approach is generating digital surface models as a spin off during the dense matching process. You can do dense matching in the same GSD as the image is available, but you have to mention, that the matching results are highly correlated with their neighbors due to the fact, that for most matching techniques the local neighborhood is used (Beckmann \& Wiedemann 2012).

\section{CONCLUSIONS}

There are a lot of quality aspects to be considered in DOP production. A qualified process chain may avoid most errors. To eliminate all errors an enormous effort is necessary. There is nowadays no way to guarantee error free orthophotos without the need of detailed manual inspections of the results.

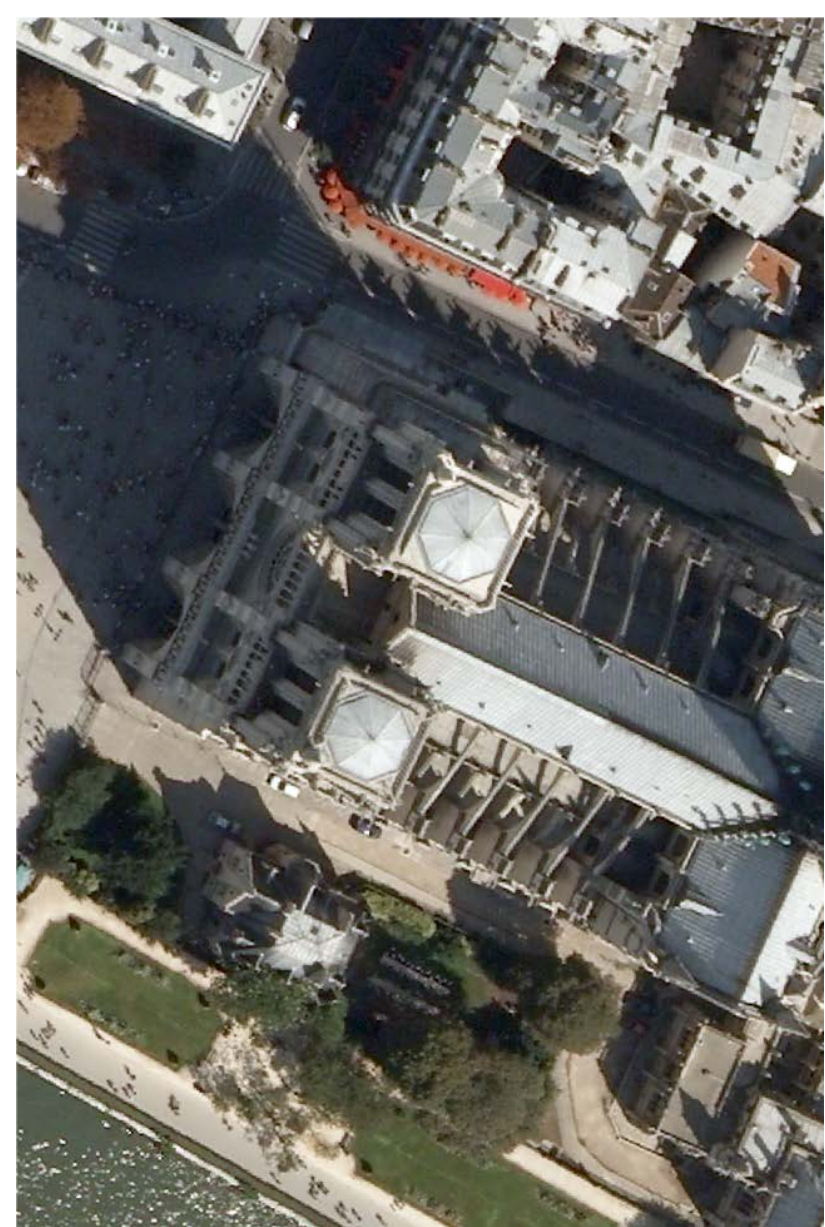

Fig. 13: Corresponding regular orthophoto

\section{REFERENCES}

AdV2012: Arbeitsgemeinschaft der Vermessungsverwaltungen der Läder der Bundesrepublik Deutschland. Produktstandard für Digitale Orthophotos. Version 1.4.

DIN 18740-3 Photogrammetrische Produkte Teil 3: Anforderungen an das Orthophoto

Wiedemann, A. \& W. Peter (2006): Combined Photogrammetric Flight with ALTM and Digital Frame Camera. In: O. Altan \& L. Gründig (eds.) Fifth Turkish-German Joint Geodetic Days. Paper presented to the conference organized at Berlin, Germany, March 28-31, on CD

Wiedemann, A. \& P. Wicki (2010): Mythos True Orthophotos Vom Sinn und Unsinn eines Produkts. Deutsche Gesellschaft für Photogrammetrie und Fernerkundung DGPF-Jahrestagung Wien 2010, Band 19

Wiedemann, A. \& S. Beckmann (2012): Dense Image Matching in der Praxis. Deutsche Gesellschaft für Photogrammetrie und Fernerkundung DGPF-Jahrestagung Potsdam 2012, Band 21 